\title{
Using Program Analysis to Improve API Learnability
}

\author{
Kyle Thayer \\ Paul G. Allen School of Computer Science \& Engineering \\ University of Washington \\ Seattle, WA \\ kthayer@cs.washington.edu
}

\begin{abstract}
Learning from API documentation and tutorials is challenging for many programmers. Improving the learnability of APIs can reduce this barrier, especially for new programmers. We will use the tools of program analysis to extract key concepts and learning dependencies from API source code, API documentation, open source code, and other online sources of information on APIs. With this information we will generate learning maps for any user-provided code snippet, and will take users through each concept used in the code snippet. Users may also navigate through the most commonly used features of an API without providing a code snippet. We also hope to extend this work to help users find the features of an API they need and also help them integrate that into their code.
\end{abstract}

\section{CCS CONCEPTS}

- Social and professional topics $\rightarrow$ Computer science education; • Software and its engineering $\rightarrow$ Software libraries and repositories;

\section{KEYWORDS}

API learnability, program analysis, auto-generated documentation

\section{ACM Reference Format:}

Kyle Thayer. 2018. Using Program Analysis to Improve API Learnability. In ICER '18: 2018 International Computing Education Research Conference, August 13-15, 2018, Espoo, Finland. ACM, New York, NY, USA, 2 pages. https://doi.org/10.1145/3230977.3231009

\section{PRELIMINARY WORK}

I am a third-year PhD student in Paul G. Allen School of Computer Science \& Engineering at the University of Washington. I have passed my Qualifying Evaluation and am now starting work toward my thesis proposal.

I have published one research project for publication: a qualitative study on barriers faced by coding bootcamp students. We found that, while bootcamps provided an alternate path into the software industry, students faced great personal costs and risks in taking this path, often including significant time, money and effort spent before, during, and after their bootcamps. To get software development jobs, students had to develop the ability to learn new

Permission to make digital or hard copies of part or all of this work for personal or classroom use is granted without fee provided that copies are not made or distributed for profit or commercial advantage and that copies bear this notice and the full citation on the first page. Copyrights for third-party components of this work must be honored.

For all other uses, contact the owner/author(s).

ICER '18, August 13-15, 2018, Espoo, Finland

(C) 2018 Copyright held by the owner/author(s).

ACM ISBN 978-1-4503-5628-2/18/08.

https://doi.org/10.1145/3230977.3231009 programming languages and libraries from documentation, tutorials and websites like StackOverflow. Many students said their bootcamp taught them this skill, though not all were happy with how this skill was taught.

I have started creating a theory of key concepts for this work, and I hope to get feedback on the scope and direction of my dissertation.

\section{BACKGROUND AND MOTIVATION}

Hearing about the challenge in using documentation, tutorials and other resources to learn new programming languages and the Application Programming Interfaces (APIs) of libraries and frameworks made us consider ways of reducing this barrier. In particular we are investigating how to use program analysis methods to create content that may help people who are learning APIs.

The challenges of using programming systems range from a programmer deciding what they want the computer to do regardless of the programming language or library, to the specific challenges of selecting programming interfaces, knowing how they work, and knowing the relevant concepts and terminology [1,2]. Using API documentation provides specific challenges as well, such as navigating documentation, understanding how API designers intended their APIs to be use, matching specific scenario needs with API features [4]. To make good decisions on what API features to use and use them properly, programmers need to learn the API they are working with.

Existing methods of learning many large APIs consist of formatted code documentation (i.e., JavaDocs), sometimes with examples and a brief intro, and human-created tutorials. The raw documentation is often difficult for newcomers to navigate and the human-created tutorials take large amounts of effort and can go out of date when new versions of APIs are released. Our previous study on coding bootcamps showed the challenge of learning APIs and how the ability to learn from API documentation and other resources are seen as a valuable skill that was difficult to acquire.

Researchers have proposed generating on-demand documentation [5] and have made various attempts to make APIs easier to learn and use. These attempts include changes to APIs designs [8], and improved methods of searching API documentation [6] and other online resources [7]. These methods focus on improving the search for specific desired features instead of on explaining user-provided code snippets.

\section{RESEARCH GOALS \& METHODS}

Given the importance of knowing relevant concepts and terminology in using APIs [2], our future research will focus on how to make it easier to learn the concepts and terminology used in arbitrary code. We will create learning maps for APIs which will be 
constructed from of key concepts and learning dependencies. By key concepts we mean the terminology, ideas, and patterns needed to perform tasks with an API, and by learning dependencies we mean the ways in which some key concepts can only be understood in relation to other key concepts.

These learning maps can be used by programmers wanting to understand code they've found or written and see how it can be expanded. They can also be used by tutorial creators in organizing their tutorials (saving time) or by newcomers as a guide for which concepts to learn (giving guidance). In particular, we believe newcomers will benefit from seeing a concise layout of key concepts which they can compare against their prior knowledge. The learning maps will provide paths to learning any key concept in an API. This will support learning one, some, or all features of the API.

In our research we will ask and attempt to answer the following research questions about key concepts, learning dependencies, and learning maps:

1) Can we extract key concepts and learning dependencies from available API code, documentation, open source repositories and question and answers sites?

To extract key concepts and learning dependencies from available code, we will first look at multiple APIs and tutorials. We will determine from the content and organization of tutorials what each one considers the key concepts of an API and what order those concepts can be presented in. These may not be the only key concepts a learner may need to know, but they will provide a baseline of concepts and APIs we will hope to recover through automated methods.

We will look at how key concepts and learning dependencies might be extracted from existing code, and other online resources, but most of the work in clarifying how to extract them will be done in conjunction with answering the next question.

Additionally, since there are many APIs, APIs change quickly, and new APIs are created, we want to use automated processes to do this work, so our second research question is:

2) Can we use program analysis to automatically extract key concepts and learning dependencies for an API?

Program analysis allows the automated extraction of features from computer programs, whether from code, execution information, or other resources. These analyses often provide information about how programs are expected to work, such as profiling, performance evaluation and bug detection [3]. We will instead use program analysis to identify key concepts and learning dependencies in APIs.

We will take the example APIs we looked at before and turn to what available code we can find for those APIs, such as: the API code, API documentation, open source code that uses the API and other resources on the API such as StackOverflow. We will then create definitions of key concepts and learning dependencies in terms of this available code and create program analyses that can extract them. As we iterate through this process we will come up with clearer definitions of our terms and better methods of automatically extracting these features.

3) How effective are learning maps generated from our automatically extracted key concepts and learning dependencies?
To test this, we will create an interface for learners that will give them a generated learning map. This interface may include automatically generated links to content for learning each concept or manually curated links for the concepts. We will then give learners tasks to complete with an API with the generated learning map, measure the effectiveness of these learning maps in terms of conceptual learning, problem solving ability, and perceived difficulty. Through this we hope to gain insights for improving to the underlying algorithms and the presentation of these learning maps.

4) Can we make learning maps based on provided code using an API?

To do this, detect which parts of an API are being used in the code and find the relevant sections of the learning map. We will then generate a tutorial that highlights concepts used in their code and also suggests possible extensions to the code.

5) Can we help developers search learning maps for the key concepts they need?

To do this, we will take user inputted searches and code, and find key concepts related to their input and their code while also considering how commonly used those concepts are. We can then help them integrate the key concepts into the code.

\section{EXPECTED CONTRIBUTIONS}

When this work has been completed, we will have created new analyses that extract newly defined factors from code bases: key concepts and learning dependencies. We will also have used these analyses to create new content and tools for API learners with specific code questions, newcomers to an API, and creators of API tutorials. Finally, we will have gained new understanding in how programmers learn APIs and what their needs are.

\section{REFERENCES}

[1] A.J. Ko, B.A. Myers, and H.H. Aung. 2004. Six Learning Barriers in End-User Programming Systems. In 2004 IEEE Symposium on Visual Languages and Human Centric Computing. 199-206. https://doi.org/10.1109/VLHCC.2004.47

[2] A. J. Ko and Y. Riche. 2011. The role of conceptual knowledge in API usability. In 2011 IEEE Symposium on Visual Languages and Human-Centric Computing (VL/HCC). 173-176. https://doi.org/10.1109/VLHCC.2011.6070395

[3] Chi-Keung Luk, Robert Cohn, Robert Muth, Harish Patil, Artur Klauser, Geoff Lowney, Steven Wallace, Vijay Janapa Reddi, and Kim Hazelwood. 2005. Pin: Building Customized Program Analysis Tools with Dynamic Instrumentation. In Proceedings of the 2005 ACM SIGPLAN Conference on Programming Language Design and Implementation (PLDI '05). ACM, New York, NY, USA, 190-200. https: //doi.org/10.1145/1065010.1065034

[4] Martin P. Robillard and Robert DeLine. 2011. A field study of API learning obstacles. Empirical Software Engineering 16, 6 (Dec. 2011), 703-732. https://doi.org/10.1007/ s10664-010-9150-8

[5] Martin P. Robillard, Andrian Marcus, Christoph Treude, Gabriele Bavota, Oscar Chaparro, Neil Ernst, Marco AurÃllio Gerosa, Michael Godfrey, Michele Lanza, Mario Linares-VÃąquez, and others. 2017. On-Demand Developer Documentation. Software Maintenance and Evolution (ICSME) (2017). http://www.inf.usi.ch/lanza/ Downloads/Robi2017a.pdf

[6] J. Stylos, A. Faulring, Z. Yang, and B. A. Myers. 2009. Improving API documentation using API usage information. In 2009 IEEE Symposium on Visual Languages and Human-Centric Computing (VL/HCC). 119-126. https://doi.org/10.1109/VLHCC. 2009.5295283

[7] J. Stylos and B. A. Myers. 2006. Mica: A Web-Search Tool for Finding API Components and Examples. In Visual Languages and Human-Centric Computing (VL/HCC'06). 195-202. https://doi.org/10.1109/VLHCC.2006.32

[8] Jeffrey Stylos and Brad A. Myers. 2008. The Implications of Method Placement on API Learnability. In Proceedings of the 16th ACM SIGSOFT International Symposium on Foundations of Software Engineering (SIGSOFT '08/FSE-16). ACM, New York, NY, USA, 105-112. https://doi.org/10.1145/1453101.1453117 